\title{
Contribution à l'étude des binaires des types F, G, K, M. VII. HD 147395, une géante $M$ binaire spectroscopique
}

\author{
Contribution to the study of F, G, K, M binaries. VII. HD 147395, a M giant \\ spectroscopic binary
}

\author{
J.M. Carquillat et N. Ginestet \\ Observatoire Midi-Pyrénées, Laboratoire d'Astrophysique de Toulouse (U.R.A. No. 285 du C.N.R.S.), 14 Avenue \\ Edouard Belin, F-31400 Toulouse, France
}

Reçu le 23 octobre; accepté le 27 novembre 1995

\begin{abstract}
HD 147395, a giant M2 star, was found to be a spectroscopic binary by the Mount Wilson astronomers some time ago, but its orbit remained unknown so far. Radial velocity observations carried out at the Observatoire de Haute-Provence with the CORAVEL spectrovelocimeter permitted us to obtain the orbital elements of this binary. They are as follows: $P=335.537$ days; $T=4448335.0 \mathrm{JD} ; \omega=336.9^{\circ} ; e=0.235 ; K=16.87 \mathrm{~km} / \mathrm{s} ; V_{0}=$ $-20.12 \mathrm{~km} / \mathrm{s} ; a_{1} \sin i=75.67 \mathrm{Gm} ; f(M)=0.154 M_{\odot}$. The spectral type of the primary, and the $f(M)$ value, lead to $M_{2} \geq 1.0 \pm 0.2 M_{\odot}$ for the mass of the unseen companion. According to the color indices $B-V$ and $U-B$ of the system, the secondary star should be a F5V. We estimate at $1.25 \pm 0.1 \mathrm{UA}$ the mean linear separation of the components; nevertheless, both the distance of this binary $(d \sim 275 \mathrm{pc})$ and the expected value of $\Delta m$ seem too important for the system to be resolved by interferometry.
\end{abstract}

Key words: stars: individual: HD 147395 — stars: spectroscopic binaries — stars: fundamental parameters

\section{Introduction}

HD 147395 (M2III; $V=6,60, B-V=1,53, U-B=1,84$ ) était signalée binaire spectroscopique dans le Catalogue général des vitesses radiales stellaires (Wilson 1953) : ce catalogue mentionne quatre observations effectuées à l'Observatoire du Mont-Wilson, et indique qu'une variation de vitesse radiale de $20 \mathrm{~km} / \mathrm{s}$ a été constatée. Pour cette raison, cet objet figurait à notre programme d'observations systématiques d'étoiles des derniers types spectraux suspectées binaires spectroscopiques.

La classification spectrale MK, ainsi que les indices de couleur indiqués plus haut proviennent d'une publication récente de Sato \& Kuji (1990) : HD 147395 a fait partie d'un programme d'observations pour la détermination de parallaxes spectroscopiques d'étoiles utilisées pour des calculs de temps et latitudes. Notons que la classification M2III obtenue par Sato et Kuji est en parfait accord avec celle, gM2, donnée précédemment dans le "Catalogue du Mont-Wilson" (Wilson 1953).

Send offprint requests to: J.M. Carquillat
Nos observations, poursuivies durant une dizaine d'années à l'Observatoire de Haute-Provence (OHP), nous ont permis la détermination des éléments orbitaux de cette binaire spectroscopique à raies simples de 11 mois de période.

\section{Obtention des vitesses radiales et des élements orbitaux}

Les observations ont été effectuées au moyen du spectrophotomètre CORAVEL (Baranne et al. 1979) monté au foyer cassegrain du télescope suisse de $1 \mathrm{~m}$ de l'OHP. Rappelons que cet appareil permet, en quelques minutes, l'obtention de la vitesse radiale (VR) d'une étoile (de type F à M) par une méthode de corrélation croisée. Trente neuf VR de HD 147395 ont ainsi été obtenues, avec une erreur standard interne moyenne de $0,25 \mathrm{~km} / \mathrm{s}$, l'observation régulière d'étoiles standards de VR (liste de l'UAI) permettant d'assurer le rattachement des mesures au système international.

Les quatre VR anciennes obtenues, à partir de spectres à $80 \AA / \mathrm{mm}$ de dispersion, par les astronomes du 
Mont-Wilson sont publiées, individuellement, dans la liste de Abt (1973), et comme elles apparaissaient compatibles avec la courbe de VR préliminaire que nous avions trouvée, nous les avons également utilisées pour la détermination de la période. L'ensemble de ces VR (MontWilson et OHP) figure en Table 1.

Tableau 1. Vitesses radiales (VR) de HD 147395 et résidus (O-C) par rapport aux vitesses calculées avec les éléments orbitaux définitifs. Les quatre premières VR, qui proviennent de l'Observatoire du Mont-Wilson, ont été utilisées seulement pour la détermination de la période

\begin{tabular}{|c|c|c|c|c|c|}
\hline $\mathrm{N}^{\circ}$ & Date TU & $\begin{array}{c}\text { Jour Jul. } \\
2400000+\end{array}$ & Phase & $\begin{array}{l}\text { V.R. } \\
\mathrm{km} / \mathrm{s}\end{array}$ & $\begin{array}{l}\mathrm{O}-\mathrm{C} \\
\mathrm{km} / \mathrm{s}\end{array}$ \\
\hline 1 & 28,52 Mars 1943 & 30812,02 & 0,776 & $-25,4$ & \\
\hline 2 & 16,45 Avr. 1946 & 31926,95 & 0,099 & $-4,6$ & \\
\hline 3 & $20,40 \mathrm{Mai}$ & 31960,90 & 0,200 & $-9,8$ & \\
\hline 4 & 18,48 Avr. 1948 & 32659,98 & 0,284 & $-18,5$ & \\
\hline 5 & 9,94 Juin 1985 & 46226,44 & 0,716 & $-30,9$ & $+0,1$ \\
\hline 6 & $14,01 \quad "$ & 46230,51 & 0,728 & $-29,8$ & $+0,6$ \\
\hline 7 & 25,80 Sept. " & 46334,30 & 0,037 & $+0,4$ & $+0,0$ \\
\hline 8 & 27,78 & 46336,28 & 0,043 & $-0,3$ & $-0,7$ \\
\hline 9 & 25,14 Fev. 1986 & 46486,64 & 0,491 & $-32,1$ & $-0,3$ \\
\hline 10 & 15,79 Oct. & 46719,29 & 0,185 & $-11,5$ & $-0,7$ \\
\hline 11 & 29,76 Oct. 1987 & 47098,26 & 0,314 & $-22,8$ & $-0,1$ \\
\hline 12 & $31,76 \quad "$ & 47100,26 & 0,320 & $-23,8$ & $-0,6$ \\
\hline 13 & 1,10 Mai 1988 & 47282,60 & 0,863 & $-18,6$ & $-0,2$ \\
\hline 14 & $5,03 \quad "$ & 47287,53 & 0,878 & $-16,2$ & $+0,3$ \\
\hline 15 & 24,77 Oct. & 47459,27 & 0,390 & $-27,2$ & $+0,4$ \\
\hline 16 & $30,74 \quad "$ & 47465,24 & 0,408 & $-28,7$ & $-0,2$ \\
\hline 17 & 16,07 Mars 1989 & 47601,57 & 0,814 & $-24,0$ & $+0,1$ \\
\hline 18 & $24,08 \quad "$ & 47609,58 & 0,838 & $-21,0$ & $+0,6$ \\
\hline 19 & 14,17 Mars 1990 & 47964,67 & 0,896 & $-14,2$ & $-0,3$ \\
\hline 20 & $18,16 \quad "$ & 47968,66 & 0,908 & $-12,1$ & $+0,1$ \\
\hline 21 & 24,80 Août " & 48128,30 & 0,384 & $-27,6$ & $-0,4$ \\
\hline 22 & $31,82 \quad " \quad "$ & 48135,32 & 0,405 & $-28,1$ & $+0,2$ \\
\hline 23 & 10,24 Jan. 1991 & 48266,74 & 0,797 & $-26,6$ & $-0,8$ \\
\hline 24 & 11,13 Mars " & 48326,63 & 0,975 & $-3,1$ & $+0,1$ \\
\hline 25 & $19,20 \quad "$ & 48334,70 & 0,999 & $-1,1$ & $-0,1$ \\
\hline 26 & 2,04 Avr. & 48348,54 & 0,040 & $+0,8$ & $+0,4$ \\
\hline 27 & 29,01 & 48375,51 & 0,121 & $-3,9$ & $+0,3$ \\
\hline 28 & 19,88 Juil. & 48457,38 & 0,365 & $-26,3$ & $-0,2$ \\
\hline 29 & 1,96 Août " & 48470,46 & 0,404 & $-28,3$ & $-0,0$ \\
\hline 30 & 10,75 Nov. & 48571,25 & 0,704 & $-31,8$ & $-0,3$ \\
\hline 31 & 18,23 Fev. 1992 & 48670,73 & 0,001 & $-0,6$ & $+0,3$ \\
\hline 32 & $21,22 \quad "$ & 48673,72 & 0,009 & $-0,4$ & $-0,0$ \\
\hline 33 & 12,04 Avr. & 48724,54 & 0,161 & $-8,4$ & $-0,1$ \\
\hline 34 & 8,07 Mai & 48750,57 & 0,238 & $-15,9$ & $+0,3$ \\
\hline 35 & 12,74 Nov. & 48939,24 & 0,801 & $-25,4$ & $+0,0$ \\
\hline 36 & 7,09 Avr. & 49084,59 & 0,234 & $-15,2$ & $+0,6$ \\
\hline 37 & 2,97 Juin & 49141,47 & 0,403 & $-28,0$ & $+0,3$ \\
\hline 38 & 10,97 Juil. & 49179,47 & 0,517 & $-32,4$ & $-0,0$ \\
\hline 39 & 17,09 Mars 1994 & 49428,59 & 0,259 & $-17,9$ & 0,3 \\
\hline 40 & $21,20 \quad "$ & 49432,70 & 0,271 & $-19,3$ & $-0,1$ \\
\hline 41 & 4,94 Juil. & 49538,44 & 0,587 & $-33,2$ & $+0,1$ \\
\hline 42 & 14,78 Oct. & 49640,28 & 0,890 & $-15,0$ & $-0,2$ \\
\hline 43 & 5,14 Mars 1995 & 49781,64 & 0,311 & $-22,3$ & $+0,2$ \\
\hline
\end{tabular}

Les éléments provisoires ont été obtenus par comparaison de la courbe préliminaire de VR avec une série de modèles de courbes, la période proche d'une année étant apparue comme la seule possibilité après quelques cycles d'observation. Le calcul des éléments définitifs a été effectué sur l'ordinateur Digital VMS 3200 de l'Observatoire de Toulouse à l'aide du programme BS1 (Nadal et al. 1979); nous avons procédé en deux temps :

1) l'ensemble des VR de la Table 1 a d'abord été traité afin d'obtenir la valeur définitive de la période orbitale, soit $P=335,537 \pm 0,036$ jours. Pour effectuer ce calcul, les mesures ont été pondérées de la manière suivante : poids 1 pour les VR obtenues à CORAVEL; poids $1 / 2$ pour les VR obtenues au Mont-Wilson.

2) en fixant la période à la valeur précédemment trouvée nous avons, pour raison d'homogénéité, déterminé les autres éléments en n'utilisant que les observations effectuées à CORAVEL.

Nous obtenons ainsi les éléments suivants :

$$
\begin{array}{rlrl}
P & =335,537 \mathrm{j} \text { (fixee) } & K=16,87 \pm 0,10 \mathrm{~km} / \mathrm{s} \\
T=4448335,0 \pm 1,2 \mathrm{JJ} & V_{0}=-20,12 \pm 0,06 \mathrm{~km} / \mathrm{s} \\
\omega & =336^{\circ}, 9 \pm 1^{\circ}, 4 & a_{1} \sin i=75,67 \mathrm{Gm} \\
e & =0,235 \pm 0,005 & & f(M)=0,154 M_{\odot}
\end{array}
$$

L'écart-type $\sigma(\mathrm{O}-\mathrm{C})$ pour une VR de poids unité est de $0,35 \mathrm{~km} / \mathrm{s}$. La courbe de VR calculée à partir de ces éléments est donnée en Fig. 1 et les résidus $\mathrm{O}-\mathrm{C}$ se trouvent à la Table 1 .

\section{Discussion}

La procédure décrite par Carquillat et al. (1982) permet, même dans le cas d'une binaire à un seul spectre visible (BS1), d'émettre des hypothèses sur la nature de l'étoile secondaire et sur la distance la séparant de l'étoile primaire. Ce calcul s'effectue à partir des équations :

$$
f(M)=M_{1} \sin ^{3} i\left(\mu^{3} /(\mu+1)^{2}\right)
$$

avec $\mu=M_{2} / M_{1}$

$$
a=a_{1} \sin i(1+1 / \mu) / \sin i
$$

il fait donc appel aux quantités déduites des éléments orbitaux $\left(f(M)\right.$ et $\left.a_{1} \sin i\right)$, mais aussi à la valeur de la masse $M_{1}$ de la primaire. Quant au facteur $\sin i$, nous avons montré, dans l'article précédemment cité, que sa valeur intervenait peu pour le calcul du demi-grand axe $a$ de l'orbite relative (séparation linéaire moyenne des composantes) tant que $i$ restait supérieur à $30^{\circ}$.

Pour ce qui concerne la masse de la primaire, nous devons en faire une estimation à partir de son type spectral MK, c'est-à-dire M2III. Schmidt-Kaler (1982) donne, pour une étoile de ce type, une masse moyenne de $1,3 M_{\odot}$ mais rapelle que, contrairement aux naines, les étoiles géantes ne constituent pas, du point de vue de leurs masses, un groupe homogène. Nous avons donc envisagé plusieurs modèles du système pour $1 M_{\odot}<M_{1}<2 M_{\odot}$. Les résultats 


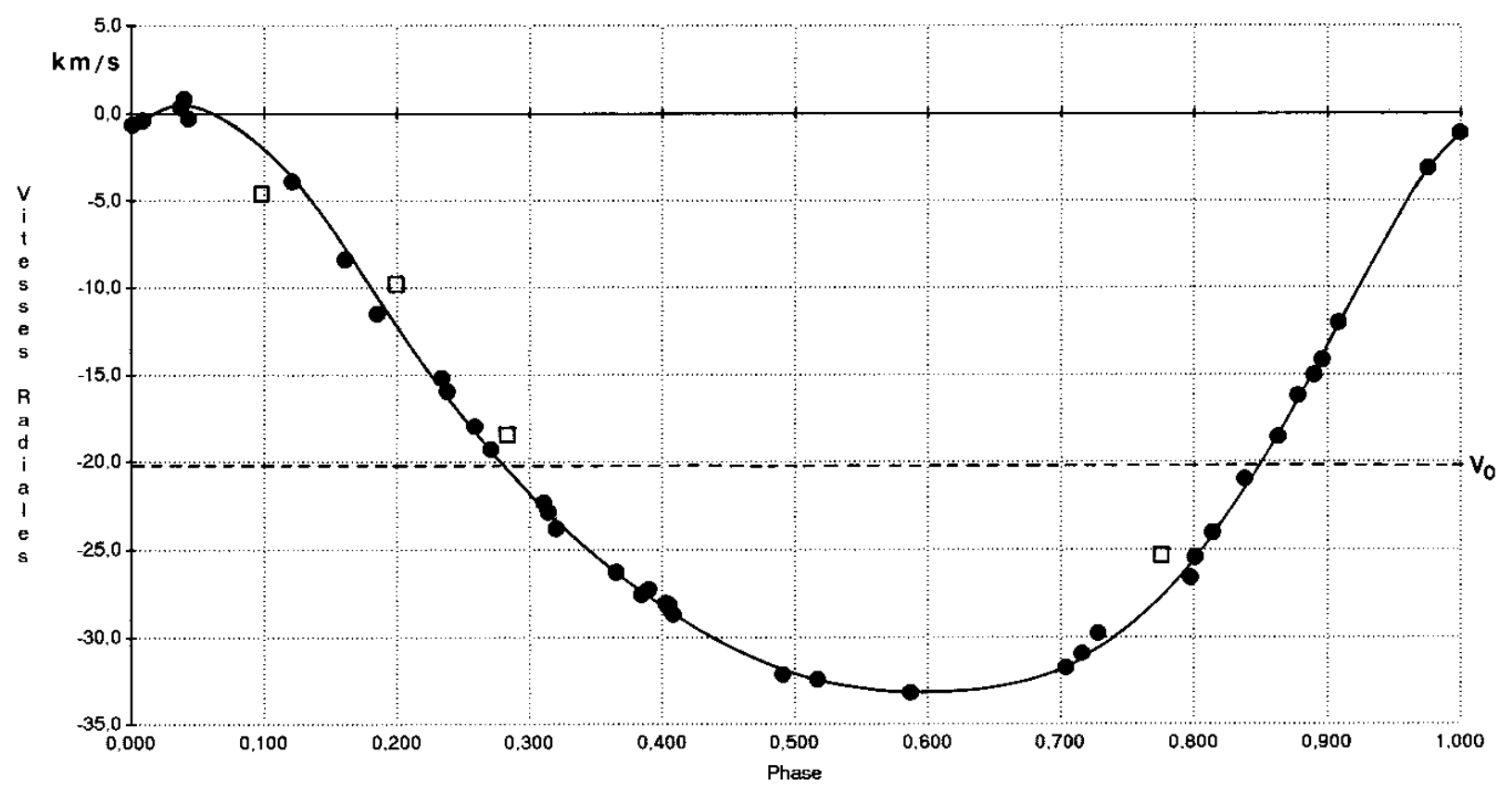

Fig. 1. Courbe de vitesse radiale de HD 147395. La phase zéro correspond au passage au périastre. Observations : • Observatoire de Haute-Provence; $\square$ Observatoire du Mont-Wilson

de cette investigation figurent dans le tableau ci-dessous :

\begin{tabular}{|l|l|l|l|c|l|}
\hline$M_{1}(\odot)$ & $\mu \min$ & $M_{2}(\odot)$ & $a(\mathrm{UA})$ & $a^{\prime \prime}$ & $i \min$ \\
\hline 1,0 & 0,79 & 0,80 & 1,14 & 0,004 & $58^{\circ}$ \\
\hline 1,5 & 0,66 & 1,00 & 1,28 & 0,005 & $48^{\circ}$ \\
\hline 2,0 & 0,58 & 1,15 & 1,37 & 0,005 & $42^{\circ}$ \\
\hline
\end{tabular}

les valeurs minimales du rapport de masses " $\mu$ min", ainsi que les valeurs de $a$, ont été obtenues en supposant $i=90^{\circ}$; les valeurs minimales de l'inclinaison orbitale " $i$ min" correspondent au cas limite $\mu=1$.

Il en résulte que :

1) la secondaire devrait avoir, approximativement, une masse minimum de $1,0 \pm 0,2 M_{\odot}$

2) la séparation linéaire moyenne des composantes peut être estimée à la valeur $a=1,25 \pm 0,1 \mathrm{UA}$.

Si l'on tient compte du fait que la trace de corrélation de la secondaire n'est pas visible avec CORAVEL, cette composante est donc vraisemblablement une étoile naine de type solaire, ou légèrement plus chaud (étoile F). En effet, l'indice de couleur $B-V=1,53$, un peu faible pour une étoile de type M2III (de même que $U-B$ ) s'accorderait bien avec une association telle M2III+F5V; la valeur de $\Delta m$, supérieure à 4 mag., est alors trop forte pour permettre l'observation du "pic" de corrélation du compagnon.

D'autre part, si nous attribuons à la primaire la magnitude absolue $M_{v}=-0,6$ correspondant à son type spectral MK (Schmidt-Kaler déjà cité), nous en déduisons que la distance du système est $d \simeq 275 \mathrm{pc}$. Compte tenu de la valeur trouvée pour $a$, la séparation angulaire des composantes sera, au maximum, de quelques millisecondes d'arc. Cette valeur est malheureusement insuffisante pour permettre de résoudre cette binaire par interférométrie des tavelures, opération qui était de toute façon compromise par la forte valeur présumée de $\Delta m$.

Remerciements. Nous tenons à remercier Michel Mayor pour l'attribution des missions d'observation à CORAVEL, et ne saurions oublier notre très regretté collègue Antoine Duquennoy qui a effectué la réduction, à l'Observatoire de Genève, des mesures de VR ayant servi de base à ce travail. Merci également aux différents collègues qui ont eu la gentillesse d'effectuer pour nous quelques observations de HD 147395 : MM. Duquennoy, Halbwachs, Imbert, North et Waelkens.

\section{Bibliographie}

Abt H.A., 1973, ApJS 26, 472

Baranne A., Mayor M., Poncet J.L., 1979, Vistas Astron. 23, 279

Carquillat J.M., Nadal R., Ginestet N., Pédoussaut A., 1982, A\&A 115, 23

Nadal R., Ginestet N., Carquillat J.M., Pédoussaut A., 1979, A\&AS 35, 203

Sato K., Kuji S., 1990, A\&AS 85, 1069

Schmidt-Kaler Th., 1982, in Landolt-Börnstein, Zahlenwerte und Funktionen aus Naturwissenschaften und Technik, Gr. 6, B. 2. Springer-Verlag, Berlin, Heidelberg, New-York, p. 1

Wilson R.E., 1953, General Catalogue of Stellar Radial Velocities, Carnegie Institution of Washington Publ. 601 